\title{
Influence of graphite morphology on static and cyclic strength of ferritic nodular cast iron
}

\author{
Christian Gebhardt $^{1 *}$, Geng Chen ${ }^{1}$, Alexander Bezold ${ }^{1}$ and Christoph Broeckmann ${ }^{1}$ \\ ${ }^{1}$ Institute for Materials Applications in Mechanical Engineering, RWTH Aachen University, 52062 Aachen, Germany
}

\begin{abstract}
High silicon alloyed nodular cast iron consists of a purely ferritic matrix and graphite nodules, mainly. Varying wall thicknesses and manufacturing conditions result in different graphite morphologies throughout a structural component. From an experimental point of view, axial fatigue and tensile tests were carried out on specimens with differently degraded graphite. From a numerical point of view, the microstructure has been modelled using a finite element (FE) approach with representative volume elements (RVE). The RVE models were built according to micrographs of fatigue specimens. The generated RVEs determine effective material properties through elasto-plastic homogenization and were subsequently analysed using a shakedown approach. In shakedown theory, the material re-enters the elastic regime after a few cycles of initial plastic deformation. This work uses the shakedown theorem to derive a lower bound estimation of the endurance limit from a non-incremental simulation. Here, the material has to be modelled elastic-perfectly plastic. The major challenge in modelling nodular cast iron is to determine suitable material parameters for the graphite and ferrite phase, revealed by parameter studies on the static and cyclic model. By using reasonable material parameters, fundamental effects, observed in the fatigue tests, were reproduced on the model level.
\end{abstract}

\section{Introduction}

Ductile cast iron is a material that meets a wide range of engineering applications due to its ductility, fatigue and corrosion properties. Since cast irons are near eutectic, they are well suited for complex and thin walled castings. As a consequence, the demand of ductile iron castings has significantly increased in the last decades, especially in sectors with extreme demands for mechanical properties such as the automotive and wind energy industry [1]. Next to classic ferritic and ferriticpearlitic ductile cast iron materials (DCI), the innovative high silicon alloyed materials GJS-500-14 and GJS-60010 gain new popularity. The materials consist of a pure ferritic matrix and mostly nodular graphite inclusions. Fatigue data is, however, rare for these materials, which restricts its use in structural applications and leads to conservative design. Some of these applications feature a varying wall thickness, which yields different graphite morphologies throughout the component. The graphite morphology influences the endurance limit of nodular cast irons [2,3]. In many industrial applications, during the design process elastic stresses are calculated using FE-analysis. Most often, fatigue design is performed by applying a single S-N-curve to the entire part using commercial software. Hence, using local endurance limits in the fatigue design process saves resources and leads to an optimised part geometry.

In general, conventional DCI lacks fatigue data in torsional and axial testing. Sofu [4] noticed that an increased number of nodules leads to higher endurance limits. Siefer [5] argues that varying microstructure does not influence the fatigue behaviour significantly. Sobota and Stets [6] stated similar theses. In circumferential bending and axial fatigue testing, the nodularity did not influence GJS-400-15 fatigue behaviour significantly. Small deviations are attributed to varying graphite shapes of the non-nodular graphite. These results contradict the findings of many other works, in which a decreasing nodularity evokes a decreasing endurance limit.

Circumferential axial bending tests of high silicon alloys GJS-450-18, GJS-500-14 and GJS-600-10 were performed by Löblich [7]. Löblich obtained small improvements of the endurance limit of silicon alloyed DCI compared to conventional GJS-500-7. However, this data is statistically secured poorly and large deviations occurred during testing. La Torre [8] detected high disparity between silicon alloyed and conventional DCI. In tensile fatigue testing, a $3.78 \% \mathrm{Si}$ alloy reached $358 \mathrm{MPa}$ fatigue limit and a low silicon alloyed DCI $170 \mathrm{MPa}$.

Describing the fatigue lifetime with models considering the microstructure is a challenging task. Three groups of empirical models stand out in the literature considering the microstructure explicitly. In fracture mechanics based models, the inclusions are considered as a crack $[9,10]$. Secondly, the graphite can be modelled as a notch in an otherwise defect free material [11, 12]. Lastly, when the graphite is considered as a defect the 
fatigue lifetime is predicted according to Murakami and De Kazinczy $[13,14]$ using the area parameter or a surrounding circle respectively. These empirical models resemble two main disadvantages. First, the assumptions may lead to errors in the fatigue lifetime predictions due to the experimental character of the model parameters. Secondly and more importantly, they fail to respond to changing boundaries, such as new micro constituents and their various shapes. Additionally, fatigue lifetime predictions depend highly on the applied loading pattern. With respect to the costs of fatigue tests and the graphite morphologies possible, a numerical methodology provides useful insights into the graphite morphology's influence on the static and cyclic strength on a model level. The approach required should account for the varying microstructure. On account of this, a suitable approach based on the representative volume element (RVE) was introduced by Hill in 1963 [15]. Over the past decades, virtual material testing using RVE models has gained wide technological and commercial significance [16]. Here, computational micro-macro mechanics determine relationships between the microstructure and the macroscopic response using models on the microscale as simple as possible, commonly referred to as RVE [17]. Appropriate boundary conditions and the size of the RVE are required to perform an adequate homogenization. The size of the RVE depends on the mechanisms investigated. An RVE can contain a single inclusion surrounded by a matrix material, often referred to as unit cell model (UCM). Apart from that, material parameters for both matrix and inclusion phase are required.

The concept of RVE modelling may be combined with cyclic models. Performing incremental FE simulations is one method to solve this task. However, the method is costly with respect to the computational power and time. Moreover, introducing and simulating the loading patterns requires great efforts. This problem is evaded using the shakedown theorem based on Melan's theorem [18], where the loading history is considered through the bounding envelope of the loading domain. Thus, the specific loading history is not required and complex loading conditions can be considered with a reasonable effort [19], which is why shakedown approaches are often referred to as direct methods (DM). Based on the condition applied, DM approaches historically ensued the static theorem by Melan or the kinematic theorem introduced by Koiter in 1956 [20]. In shakedown theory, the structure deforms plastically in the first few cycles. Then, the material returns to the elastic regime and behaves pure elastically in subsequent cycles. Mathematically, all conditions of the theorem form an optimisation problem whose result is a so-called load factor, which leads to a lower bound estimation of the endurance limit of the structure. In the original theorem, the application is restricted to elastic-perfectly plastic material.

In modelling nodular cast iron, the choice of appropriate material parameters is the major challenge, especially when the phases are modelled elastic or elastic-perfectly plastic. Material parameters for the matrix phase are found easily compared to the graphite phase. Here, comparing grain size and chemical composition to single phase materials is one possible way. A matrix equivalent material was casted in [21]. Uniaxial tension tests at different temperatures and nominal strain rates provide material parameters subsequently used in micromechanical simulations. The graphite phase, however, shows strong anisotropic behaviour, indicated by nanoindentation tests performed in [22]. There, indentations on varying graphite shapes yielded a high diversity of material responses. The issues when measuring elastic properties of particles in composites were investigated in [23]. Resultant, the Oliver-Pharr method cannot be applied to accurately determine the elastic modulus, especially when the indentation depth is not within a particle-dominated depth. Therefore, a high scatter of Young's moduli and yield strength for the graphite are reported in the literature, resulting likewise partly from nanoindentation tests $[24,25]$. Other values for the Young's modulus of the graphite nodules result from assumptions made and results obtained in micromechanical simulations. Early studies [26] modelled the graphite as voids because a single phased graphite material has a negligible stiffness. An early debonding observed in uniaxial tension tests supports this assumption. Several studies investigate DCI with unit cell models [27-33]. These models are suitable to investigate material parameters and models. They are oversimplified when graphite morphologies should be investigated. RVE models obtained directly according to micrographs are shown in $[24,34-36]$. Lastly, a 3D UCM of a single inclusion has been developed in [37] by performing metallographic investigation of up to 100 slices of the same cross section. Assembling the micrographs yielded a model of a single nodule. Summarizing the literature, accurate material parameters for the graphite phase are hard to find. The Young's modulus varies considerably from $0-375 \mathrm{GPa}$, pointed out by [28].

\section{Experimental Procedure}

\subsection{Fatigue Testing}

The setup for the casting of the raw material consist of 12 cuboids $(100 \times 200 \mathrm{~mm})$. A single sprue is used in the middle of the setup. Each cuboid has a single riser. Each cuboid yields four specimens, manufactured in vertical direction from the raw material. Varying the magnesium and cerium content spawned four different graphite morphologies. The first alloy $R$ is taken as reference due to its high nodularity. A graphite morphology with reduced nodularity is called $R N$. The abbreviation $B N$ indicates bigger nodules. Finally, $V$ refers to a vermicular-like graphite morphology. Thermal analysis determined the carbon content. Spectroscopy provided silicon-, magnesium and cerium content, illustrated in Tab. 1.

Table 1. Chemical composition of investigated alloys in wt $\%$.

\begin{tabular}{ccccc} 
& $\mathrm{R}$ & $\mathrm{RN}$ & $\mathrm{BN}$ & $\mathrm{V}$ \\
\hline $\mathrm{C}$ & 3,02 & 3,07 & 3,00 & 3,07
\end{tabular}




\begin{tabular}{lcccc}
$\mathrm{Si}$ & 3,78 & 3,85 & 3,95 & 3,95 \\
$\mathrm{Ce}$ & $<0,0033$ & $<0,0033$ & 0,0470 & $<0,0033$ \\
$\mathrm{Mg}$ & 0,0327 & 0,0280 & 0,0416 & 0,0218 \\
\hline
\end{tabular}

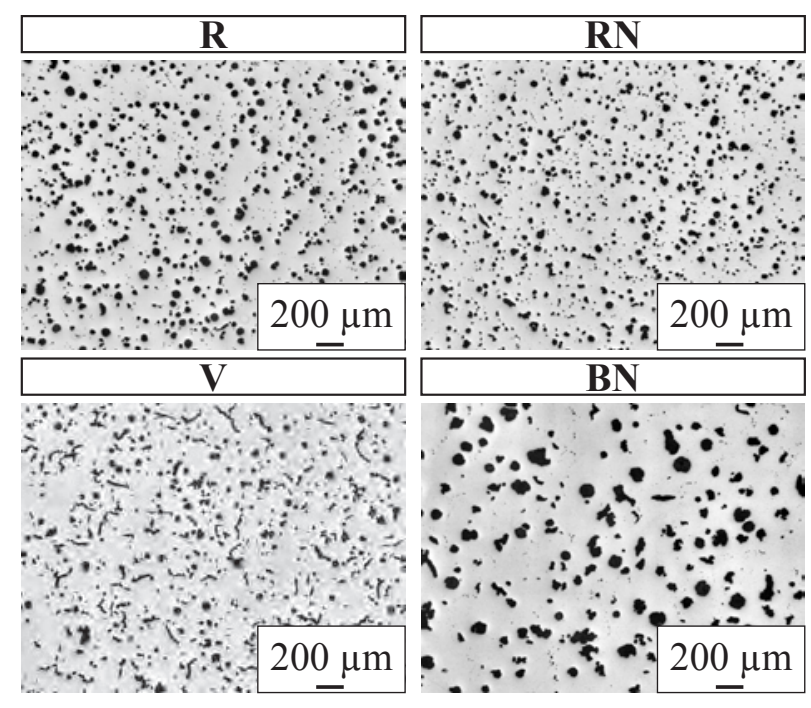

Fig. 1. Representative graphite morphologies: reference (R), reduced nodularity $(\mathrm{RN})$, vermicular $(\mathrm{V})$ and bigger nodules (BN).

The carbon- and silicon contents do not vary significantly, whereas the cerium and magnesium contents are high for the material with bigger nodules $B N$. The magnesium content is slightly elevated for $\mathrm{R}$ leading to bigger nodules compared to $R N$. Micrographs ensured the specimens taken from the blocks have a constant graphite morphology. The fatigue specimens were drilled and axial polished to a diameter of $12 \mathrm{~mm}$. The length of the specimen is $200 \mathrm{~mm}$. Axial fatigue testing was performed on electronically modernized $100 \mathrm{kN}$ Amsler HFP 422 pulsator fatigue testing machines using a stress ratio $R=0$. A crack is detected on a frequency drop of $5 \mathrm{~Hz}$. The testing procedure applied is described in [38]. The endurance limit is obtained with a stair-case procedure using at least 15 specimens. An additional amount of 5 specimens is then tested on the highest stress level to statistically secure the transition region to long life fatigue. At most, this method saves number of specimens. No additional information is adversely obtained when few fractures occur on the highest stress level. The fatigue tests stop at $N=10^{7}$ loading cycles.

\subsection{Uniaxial tension tests}

Tensile tests were carried out at room temperature using cylindrical specimens. The specimens were machined from the cast samples with a diameter of $10 \mathrm{~mm}$ and $58 \mathrm{~mm}$ of gauge length according to the DIN 50125-B. An amount of eight specimens was tested for each alloy $R, R N, V$, and $B N$. The specimens of $R, R N$ and $V$ were manufactured using material from the same batch as the fatigue specimens, whereas the specimens for $B N$ were machined from a comparable alloy with comparable graphite morphology. Metallographic samples of an elastically deformed region (A), a mostly elastically deformed region (B) and a fully plastic region of a tensile specimen are shown in Fig 2. The frequency of debonded nodules increases with increasing plastic deformation. It has to be noted, that decohesion takes place already in the almost elastically deformed region (A). Besides the tensile tests, this effect could also be attributed to grinding during preparation of the metallographic specimens. Nevertheless, the micrographs reveal a weak connection between the two phases.

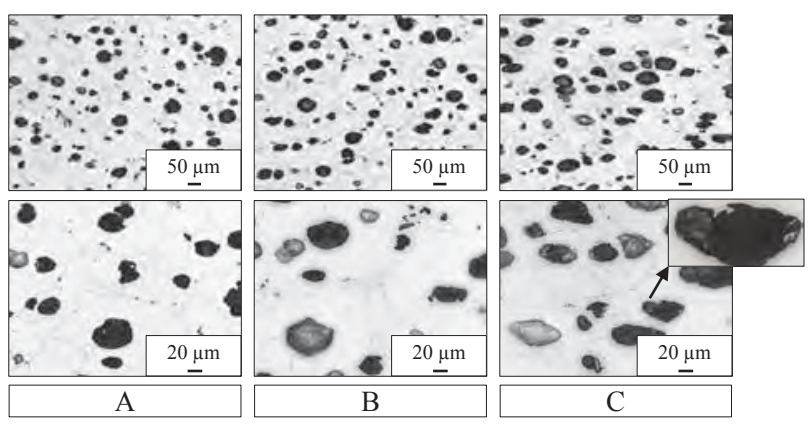

Fig. 2. Elastic (A) and plastic (B, C) deformation GJS-500-14 in a uniaxial tension test.

\section{Numerical Modelling}

\subsection{Model}

The RVE-Models are built directly according to binarized micrographs, where the pixels are classified according to Otsu's method. To determine a magnification that represents all geometrical features of the microstructure, the same area is analysed using $50 \mathrm{x}$, $100 \mathrm{x}$ and 200x magnification. A single pixel of a micrograph taken with 50x magnification covers an area of $4,19 \mu \mathrm{m}^{2}$. Thus over- or underestimates the graphite area depending on the threshold used to classify the pixels. The graphite area of the $100 \mathrm{x}-$ and $200 \mathrm{x}$ magnification is approximately equal. However, large scatter is obtained comparing single micrographs of the 200x magnification. Therefore, the size of a 200x magnification is not representative. Consequently, the RVE is built directly from a micrograph of $100 \mathrm{x}$ magnification. Beyond the graphite area, shape parameters such as circularity, aspect ratio etc. are analysed. The metallographic specimens were ground and polished. The microscope used is AxioImager M2m Zeiss with the camera ProgRes SpeedXTcore5 Jenoptik. A 2.5D RVE-Model is built according to [39], as the complexity of the microstructure prohibits the use of 3D RVE. The $2.5 \mathrm{D}$ approach is a compromise between plane stress and strain approach and is not mesh sensitive compared to a conventional 2D approach. The algorithm compares positions of the binarized micrograph to an initial hexahedral mesh. The graphite inclusions are identified, sketched and remeshed using wedge elements. This methodology allows more DOF in mesh generation compared to substituting techniques. A convergence analysis shows that a hexahedral mesh converges slightly faster while a relative error of $0.99 \%$ compared to the tetrahedral mesh occurs in the converged state. The edge length of elements is $3 \mu \mathrm{m}$ 
leading to $\sim 250.000$ elements per RVE-Model. After the 2D-Meshing, the model is extruded to $2.5 \mathrm{D}$ in Hypermesh. Multiple-point constraints introduce the boundary conditions respectively. Using the shakedown model presented in [40], unfavourable simplifications are required. Both phases are elastic-perfectly plastic. Additionally, the graphite and ferrite phase are modelled perfectly bonded.

Table 2. Summary of the RVE-model.

\begin{tabular}{lr}
\hline Micrograph & $100 \mathrm{x}$ \\
Type & $2.5 \mathrm{D}$ \\
Size & $1 \mathrm{~mm} \times 1 \mathrm{~mm} \times 1 \mu \mathrm{m}$ \\
Element type & $\mathrm{C} 3 \mathrm{D} 6$ \\
Elements quantity & $\sim 250.000$ \\
Ferrite / graphite & Elastic-perfectly plastic \\
\hline
\end{tabular}

\subsubsection{Elasto-plastic homogenisation}

The relations

$\Sigma=\frac{1}{\Omega} \int \boldsymbol{\sigma} d \Omega$

$\boldsymbol{E}=\frac{1}{\Omega} \int \boldsymbol{\varepsilon} d \Omega$

hold for small deformations in absence of body forces and perfectly bonded phases relating the microscopic stresses $\boldsymbol{\sigma}$ and strains $\boldsymbol{\varepsilon}$ to the macroscopic stress $\boldsymbol{\Sigma}$ and strain $\mathbf{E}$ tensors on the RVE-domain $\Omega$. The equations are referred to as average stress- and strain theorems respectively. When all phases are elastic and perfectly bonded

$\boldsymbol{\Sigma}=\bar{C}: \boldsymbol{E}$

holds and the effective Young's modulus is taken directly from the effective elastic tensor $\bar{C}$. In plasticity, the micro strains are additively decomposed into an elastic and plastic part:

$\varepsilon(\mathbf{u})=\varepsilon^{e}+\varepsilon^{p}$

Suquet [41] proposed a macroscopic strain measure

$\boldsymbol{E}^{p}=\boldsymbol{E}-\overline{\mathrm{C}}^{-1}: \boldsymbol{\Sigma}$

which is used to get the macroscopic yield strength defined as follows :

$\Sigma_{Y}=\arg \left(E_{e q}^{p}\left(\Sigma_{e q}\right)=0.1 \%\right)$

Kinematic uniform boundary conditions in two independent directions are imposed. Pierard [42] showed for a sufficient size of the RVE, the macroscopic response is independent from its chosen boundary condition which was ensured for the model.

\subsubsection{Shakedown model}

The static shakedown theorem by Melan is used to determine the shakedown factor $\alpha$. The materials are assumed to be Mises-type, therefore the existence of a convex yield function $f\left(\boldsymbol{\sigma}(\boldsymbol{x}, t), \sigma_{Y}\right)$ is assumed. The deformation is restricted to elastic-perfectly plastic. According to [43] when shakedown happens the total plastic dissipation is bounded, therefore the plastic strain $\dot{\varepsilon}^{p}$ would stop developing. Consequently, a timeindependent residual stress field $\overline{\boldsymbol{\rho}}$ exists and the total stress can be expressed as follows:

$\boldsymbol{\sigma}(\boldsymbol{x}, t)=\alpha \boldsymbol{\sigma}^{E}(\boldsymbol{x}, t)+\overline{\boldsymbol{\rho}}(\boldsymbol{x}, t)$

The index $E$ denotes the stress field in a purely elastic reference body. Furthermore, König showed that for materials with a convex yield function the material will shake down over any given load path within a load domain. Then Melan's shakedown theorem is given as

$$
\begin{array}{cl}
\max _{\alpha, \overline{\boldsymbol{\rho}}} & \alpha \\
\text { subject to } & \boldsymbol{\nabla} \cdot \overline{\boldsymbol{\rho}}=\mathbf{0} \text { in } \Omega \\
& \overline{\boldsymbol{\rho}} \cdot \boldsymbol{n}=\mathbf{0} \text { on } \Gamma_{1} \\
& F\left(\alpha \boldsymbol{\sigma}^{E}\left(\boldsymbol{P}_{k}\right)+\overline{\boldsymbol{\rho}}\right) \leq \sigma_{Y}^{2}
\end{array}
$$

The static shakedown theorem is expressed in finite element formulation as:

$$
\begin{array}{ll}
\min _{\alpha, \overline{\boldsymbol{\rho}}} & -\alpha \\
\text { subject to } & \sum_{i=1}^{N G} \boldsymbol{C}_{i} \boldsymbol{\rho}_{i}=\mathbf{0} \\
& F\left(\alpha \boldsymbol{\sigma}_{i k}^{e}+\overline{\boldsymbol{\rho}}_{i}\right)-\sigma_{Y, i}^{2} \leq 0
\end{array}
$$

The matrix $\boldsymbol{C}$ takes the role of the gradient-operator for $k$ load vertices and $i$ Gaussian points. The problem is reformulated according to [44] for numerical reasons. The RVEs are modelled as previously explained. The loadings are introduced through static variables, meaning forces or stresses. Two independent uniaxial loadings are considered. The elastic stress fields are calculated using the commercial FE-solver Abaqus. The lower vertex $\boldsymbol{P}$ is zero respectively, corresponding to a pulsating cyclic loading. The optimisation problem is then formulated using an in-house Matlab based code. The problem is solved by the commercial optimisation toolbox Gurobi.

\subsection{Parametric studies}

\subsubsection{Static model}

The procedure previously mentioned is used to model a micrograph from a uniaxial tension test. The goal of this study is to understand the influence of the material parameters on a model level to clarify the uncertainty about the material parameters, pointed out in the literature review. Thus, the material parameters for the elastic-perfectly plastic phases vary systematically, as illustrated in Tab. 3. The Young's modulus for the matrix phase is estimated based on its silicon content and an empirical relation given in [45]. 
Table 3. Parametric study of static model.

\begin{tabular}{lcc}
\hline \multicolumn{1}{c}{ Parameter } & Ferrite & Graphite \\
\hline Young's modulus $E[\mathrm{MPa}]$ & 206660 & $1-300000$ \\
Yield strength [MPa] & $350-1000$ & $1-1000$ \\
\hline
\end{tabular}

The phases are perfectly bonded. Consequently, the model is highly idealistic with respect to the results of the uniaxial tension tests. Regardless, the frequency of debonded nodules is not high and as the macroscopic Young's modulus and the macroscopic yield strength are investigated, the effect of decohesion is neglected. The admissible range of the macroscopic Young's modulus is set to $160.000 \pm 5.000 \mathrm{MPa}$. Furthermore, the stressstrain curve obtained from the simulation is compared to an experimental curve until $1 \%$ macroscopic plastic strain using squared errors. Based on these criteria, the red markers in Fig 3 indicate admissible sets of material parameters for an elasto-plastic model of idealised nodular cast iron.

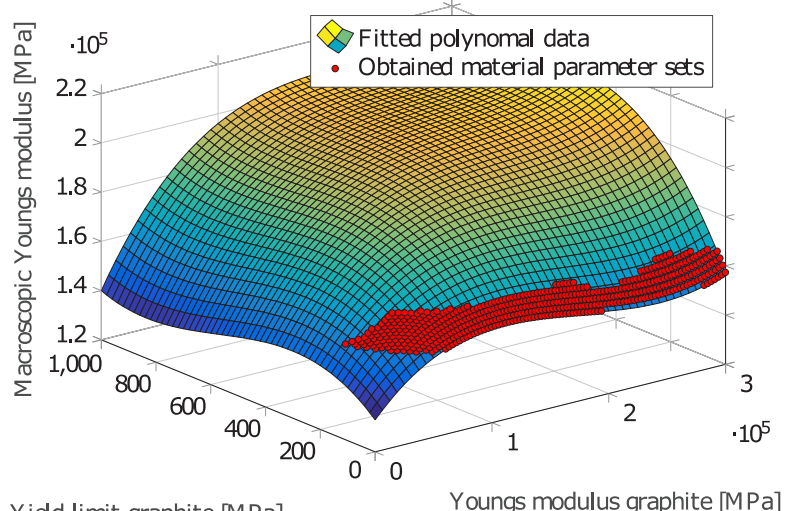

Yield limit graphite [MPa]

Youngs modulus graphite [MPa]

Fig. 3. Exemplary parameter study for a matrix yielding of $600 \mathrm{MPa}$.

None of the values from the literature for the graphite Young's modulus can be excluded. Moreover, multiple combinations of graphite Young's modulus $(1 \mathrm{MPa}-$ $300 \mathrm{GPa})$ and yield strength $(1-172.5 \mathrm{MPa})$ are possible. Here, high values of one quantity correlate with low values of the other and vice versa. Applying the balance of mechanical energy, the external applied energy density splits into an averaged strain energy density and an averaged dissipation density. By further splitting the microstress into a self-equilibrated residual stress field and an elastic stress occurring in a purely elastic reference body, the average strain energy density separates in three parts. The applied external energy density is partly globally reversible, partly stored in residual strains and partly dissipated. Manipulating the material parameters of the elasto-plastic phases will influence the macroscopic response enabling multiple possible sets of material parameters.

\subsubsection{Shakedown model}

Two parametric study were carried out on the shakedown model to understand the influence of the graphite's material parameters under cyclic loads (Tab. 4). First, a study with a small step size was taken out in the low graphite Young's modulus range. Secondly, the influence of the graphite material parameters was studied in the high graphite Young's modulus range.

Table 4. Parametric study of cyclic model.

\begin{tabular}{lcc}
\hline \multicolumn{1}{c}{ Parameter } & Ferrite & Graphite \\
\hline Young's modulus $E[\mathrm{MPa}]$ & 206660 & $1-300000$ \\
Yield strength $[\mathrm{MPa}]$ & 600 & $1-170$ \\
\hline
\end{tabular}

The material parameters of the matrix phase remain constant. In Fig. 4, the data points are linearly interconnected for constant values of the graphite yield strength $\sigma_{Y, G}$. Close to a graphite Young's modulus of $0 \mathrm{MPa}$, all curves tend towards a unique value. Afterwards, the shakedown load converges. At roughly $20,000 \mathrm{MPa}$ no significant change on the shakedown load occurs, suggesting it became independent from the graphite Young's modulus.

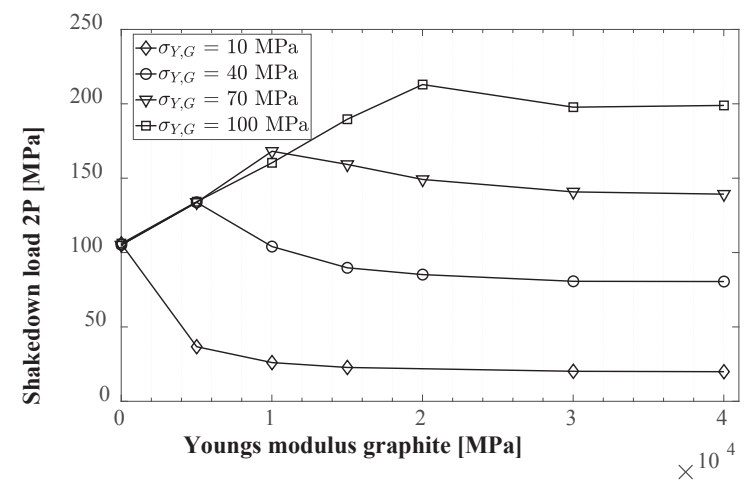

Fig. 4. Influence of low graphite Young's modulus on macroscopic shakedown load.

Fig. 5 reveals this assumption is hardly true for graphite Young's moduli higher than approximately $150,000 \mathrm{MPa}$. Why the shakedown load is independent from the Young's modulus in this range requires further investigation. A conceivable explanation reads: The location of failure changes from the nodules to the matrix when the curves become stable at roughly 20,000 MPa in Fig 4. Consequently, the shakedown limit drops due to an increase of the Young's modulus and vice versa. When the stiffness becomes sufficiently high, it induces high local stresses in the matrix. The deviatoric parts of the stress intuitively favour the failure of the matrix. 


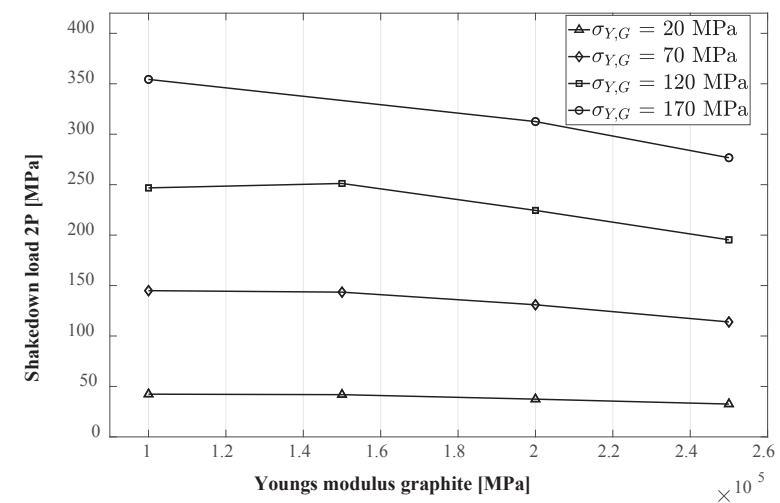

Fig. 5. Influence of high graphite Young's modulus on macroscopic shakedown load.

\section{Results and Discussion}

\subsection{Experimental investigation- Endurance limit and tensile strength}

The testing is statistically analysed with SAFD software [46]. A combined procedure is applied, where the stair step procedure yields a data point for $50 \%$ failure probability. All stress levels with more than five specimens provide additional data points in the regression of the stress amplitude over the failure probability. The data is weighted with respect to the number of specimen. Tab. 5 shows the pulsating fatigue strength $\sigma_{A, R=0}$ for the investigated alloys with a failure probability of $50 \%$.

Table 5. Results of fatigue and tensile testing and microstructural analysis.

\begin{tabular}{|c|c|c|c|c|}
\hline & $\mathrm{R}$ & $\mathrm{RN}$ & $\mathrm{BN}$ & $\mathrm{V}$ \\
\hline$\sigma_{A, R=0, P=50 \%[\mathrm{MPa}]}$ & 148.9 & 156.3 & 153.2 & 137.8 \\
\hline $\begin{array}{l}\text { Ultimate Strength } R_{m} \\
\text { [MPa] }\end{array}$ & 536 & 528 & 535 & 487 \\
\hline $\begin{array}{l}\text { Nodularity } \\
\text { DIN 459-4 [\%] }\end{array}$ & 82.7 & 70.5 & 76.7 & 46.9 \\
\hline $\begin{array}{l}\text { Nodularity } \\
\text { ASTM E2567 [\%] }\end{array}$ & 72.0 & 54.3 & 51.8 & 33.0 \\
\hline $\begin{array}{l}\text { Nodule Area (Avg / StD) } \\
{\left[\mu \mathrm{m}^{2}\right]}\end{array}$ & $\begin{array}{r}738 / \\
859\end{array}$ & $\begin{array}{r}546 / \\
659\end{array}$ & $\begin{array}{r}1860 \\
/ 264 \\
9\end{array}$ & $\begin{array}{r}680 / \\
951\end{array}$ \\
\hline Nodule Density $\left[1 / \mathrm{mm}^{2}\right]$ & 163 & 176 & 63 & 143 \\
\hline $\begin{array}{l}\text { Max Feret (Avg / StD) } \\
{[\mu \mathrm{m}]}\end{array}$ & $\begin{array}{r}33.0 / \\
20.7\end{array}$ & $\begin{array}{r}30.5 / \\
18.7\end{array}$ & $\begin{array}{r}50.6 / \\
43.6\end{array}$ & $\begin{array}{r}38.3 / \\
31.4\end{array}$ \\
\hline $\begin{array}{l}\text { Min Feret (Avg / StD) } \\
{[\mu \mathrm{m}]}\end{array}$ & $\begin{array}{r}23.9 / \\
15.7\end{array}$ & $\begin{array}{r}21.0 / \\
12.8\end{array}$ & $\begin{array}{r}33.5 / \\
29.9\end{array}$ & $\begin{array}{r}22.3 / \\
16.0\end{array}$ \\
\hline
\end{tabular}

The transition region was obtained for $R, R N$ and $V$. Too many runouts prohibited an analysis of the transition region in the $B N$-series. Comparing the endurable stress amplitudes, vermicular graphite decreases the endurance limit, while there is no significant difference between the reference graphite, the graphite with reduced nodularity and the bigger nodules. Exemplary, fig. 6 shows a statistical evaluation of the fatigue testing for the vermicular-like graphite morphology $V$. The combined procedure yields three points for the stress levels and an additional point resulting from the stair-case procedure. The good correlation of the stress amplitude with the failure probability has a square error of $R=0.9681$. The ultimate strength of $R, R N$, and $B N$ shows no significant differences, whereas a reduction is obtained for the vermicular-like graphite morphology. Additional metallographic samples were taken from fatigue testing specimens of each series. The microstructure was analysed using an in-house MatLab code. Nodules with an area greater than $30 \mu^{2}$ were considered. The nodularity was analysed according to the standard ASTM E2567. Additionally, the bounds from Velichko [47] were used to classify the nodules into graphite types and determine the nodularity according to standards DIN 459-4. The nodularity is the highest for the reference $R$. $R N$ and $B N$ have values of $70.6 \%$ and $76.7 \%$ respectively. The nodularity is low for the vermicular like graphite $V$ with a value of $46.9 \%$. The nodularity calculated according to the ASTM standard deviates significantly from those calculated with the DIN standard. The nodule density is high for the $R N$ morphology where the endurance limit is also the highest. As the $B N$-series has mostly bigger nodules compared to the others, the nodule density is low with 63 $1 / \mathrm{mm}^{2}$.

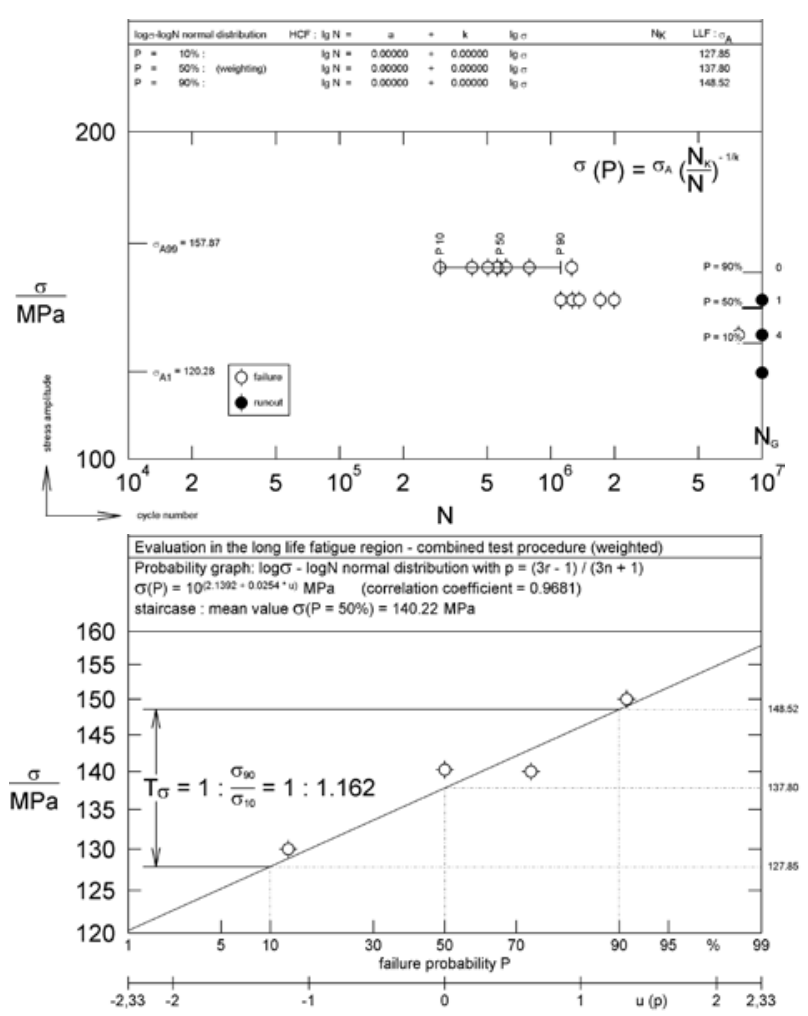

Fig. 6. Exemplary statistical evaluation of the fatigue testing for the vermicular-like graphite morphology $V$.

\subsection{Shakedown}

An amount of eleven micrographs per graphite morphology $R, R N, B N$ and $V$ was taken from specimens 
of the fatigue testing to model the RVEs. The matrix yield strength was set to $600 \mathrm{MPa}$ and the Young's modulus to 206,660 MPa. A graphite Young's modulus of $15 \mathrm{GPa}$ and a yield strength of $40 \mathrm{MPa}$ are chosen because here, failure is expected to occur in the matrix and the macroscopic response is reasonable with respect to the parametric study. The highest relative error between two loading directions is $1.1 \%$. Therefore, the data points in the boxplots in Fig. 7 are mean values respectively.

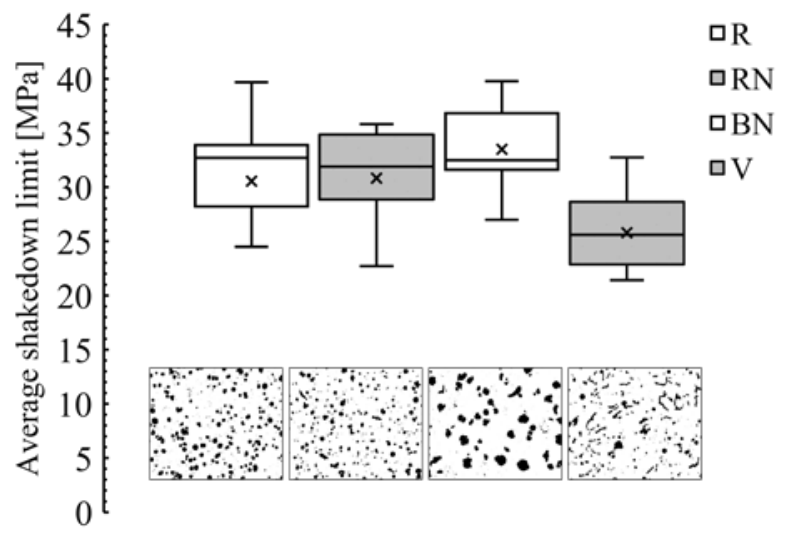

Fig. 7. Boxplots of averaged shakedown limits. Eleven micrographs were analysed per sample group.

The vertical lines mark the maximum and minimum values of a sample group. A single RVE in the R-series was excluded as the value was outside of the 1.5 interquartile range. Here, a single extremely degraded nodule resulted in a low shakedown limit of 15.4 MPa. The average shakedown limit for the sample group $V$ is the lowest, corresponding to the experiment. High deviatoric stress parts occur at the graphite phase. Intuitively, high deviatoric parts decrease the shakedown limit as they contribute to the Mises stress. The sample groups $R, R N$ and $B N$ are higher with small differences between the groups. The shakedown limits of the bigger nodules seem surprising with respect to defect based approaches. However, as the nodule density is low the equality constraints are fulfilled easier. Moreover, deviatoric stress merely occurs on the microscale as the shape of the nodules is mostly round. Comparing the grain sizes of the investigated alloys, assuming a constant yield strength for the matrix does not seem reasonable. The scatter within a sample group is considerably high and is attributed to high geometrical variance of the microstructure within the groups. Consequently, the microstructure should be evaluated on a statistical basis with a large number of samples.

\section{Conclusion}

This work compared four different graphite morphologies on an experimental and numerical basis. The experiments reveal that defect-based approaches might be unsuitable for estimating the fatigue limit of high silicon alloyed nodular cast iron, as bigger nodules had a higher fatigue limit. As expected, vermicular graphite decreases the fatigue limit. However, the fatigue limit does not correlate with the nodularity. Broadly speaking, DCIs with round nodules favour a higher lifetime independent from the nodule's size. When the graphite is degraded, the size controls the fatigue limit. However, it is difficult to draw conclusions about the graphite morphology because, in fact, four different alloys were investigated.

The core issue in modelling nodular cast iron is to define suitable material parameters for the graphite and ferrite phase. A set of material parameters was presumed where the failure seems to take place in the matrix according to the experimental observations. Fundamental effects of the graphite morphology were reproduced using the shakedown theorem on simplified RVE models obtained from micrographs of the fatigue specimens. The values are not comparable to the experimental endurance limit due to various reasons, primary because the shakedown limit does not correspond to a failure probability of $50 \%$. The high values for the $B N$ graphite morphology qualitatively agree with the results of the experiments. This is attributed to the high hydrostatic parts, which do not contribute to the Mises stress. However, effects of the matrix were completely neglected and hardening was not considered. The matrix yield strength depends on the grain size and therefore on the graphite morphology.

\section{Acknowledgements}

The project has been funded through the German ministry for economy and technology (BMWi) by a resolution of the German Bundestag. IGF 18524N

\section{References}

1. $\quad$ N.S. Tiedje, MST 26, 505-514 (2013). doi:

10.1179/026708310X12668415533649

2. N. Costa, N. Machado, F.S. Silva, CTMAT 20, 120-128 (2008)

3. M. Endo, JSMS 38, 1139-1144 (1989). doi: 10.2472/jsms.38.1139

4. M. Sofu, S. Okada, A. Sobota, AFS Transaction, 173-182 (1978)

5. W. Siefer, K. Orths, Gießerei-Forschung 4, 3-7 (1969)

6. W: Stets, A. Sobota, (AiF Nr. 13507 N/1)

7. H. Löblich, (AiF Nr. 41 EN, 2012)

8. U. de La Torre, A. Loizaga, J. Lacaze, J. Sertucha, MST 30, 1425-1431 (2013). doi: 10.1179/1743284713Y.0000000483

9. M.H. El Haddad, T.H. Topper, K.N. Smith, EFM 11, 573-584 (1979). doi: 10.1016/00137944(79)90081-X

10. N. Costa, N. Machado, F.S. Silva, Int. J. Fatigue 32, 988-995 (2010)

11. P. Lukas, L. Kunz, B. Weiss, R. Stickler, FFEMS 3, 195-204 (1980)

12. M.R. Mitchell, J. Eng. Mater. Technol. 99, 329 (1977). doi: 10.1115/1.3443549

13. F. de Kazinscky, J. Iron Steel Inst. 20, 851-855 (1970)

14. Y. Murakami, S. Nemat-Nasser, EFM 16, 373-386 (1982). doi: 10.1016/0013-7944(82)90115-1

15. R. Hill, J. Mech. Phys. Solids 11 357-372 (1963). doi: 10.1016/0022-5096(63)90036-X 
16. J. Llorca, C. González, J.M. Molina-Aldareguía, J. Segurado, R. Seltzer, F. Sket, M. Rodríguez, S. Sádaba, R. Muñoz, L.P. Canal, Advanced materials (Deerfield Beach, Fla.) 23, 130-147 (2011). doi: 10.1002/adma.201101683

17. S.J. Hollister, N. Kikuchi. Comput. Mech. 10, 7395 (1992). doi: 10.1007/BF00369853

18. E. Melan, Der Spannungszustand eines Mises Henckyschen Kontinuums bei veränderlicher Belastung. (Hölder-Pichler-Tempsky; Akad. d. Wiss, Wien, Leipzig, 1938)

19. J.W. Simon, D. Weichert, Comput. Mech. 49, 477485 (2012). doi: 10.1007/s00466-011-0656-8

20. W.T. Koiter: KNAW 14-34 (1956)

21. T. Andriollo, K. Hellström, M.R. Sonne, J. Thorborg, N. Tiedje, J. Hattel, J. Mech. Phys. Solids 111 333-357 (2018). doi: 10.1016/j.jmps.2017.11.005

22. S.K. Pradhan, B.B. Nayak, S.S. Sahay, B.K. Mishra, Carbon 47, 2290-2292 (2009). doi: 10.1016/j.carbon.2009.04.024

23. W. Yan, C.L. Pun, Z. Wu, G.P. Simon, Composites Part B: Engineering 42, 2093-2097 (2011). doi: 10.1016/j.compositesb.2011.05.002

24. D.O. Fernandino, A.P. Cisilino, S. Toro, P.J. Sanchez, Int. J. Fract 207, 1-26 (2017). doi: 10.1007/s10704-017-0215-1

25. P. Dierickx, C. Verdu, A. Reynaud, R. Fougeres, Scripta Materialia 34, 261-268 (1996). doi: 10.1016/1359-6462(95)00496-3

26. S.H. Pundale, R.J. Rogers, G.R. Nadkarni, AFS Transactions 106, 99-106 (1998)

27. T. Andriollo, J. Thorborg, N.S. Tiedje, J. Hattel, Mater. Sci. Eng. 84, 12-27 (2015). doi: 10.1088/1757-899X/84/1/012027

28. T. Andriollo, J. Hattel, Mech. Mat. 96, 138-150 (2016). doi: 10.1016/j.mechmat.2016.02.007

29. T. Andriollo, J. Thorborg, J. Hattel, Int. J. Solid. Struct. 100-101, 523-535 (2016). doi: 10.1016/j.ijsolstr.2016.09.023

30. N. Bonora, A. Ruggiero, Int. J. Solid. Struct. 42, 1401-1424 (2005). doi: 10.1016/j.ijsolstr.2004.07.025

31. W. Brocks, S. Hao, D. Steglich, J. Phys. IV France 6, 43- 52 (1996). doi: 10.1051/jp4:1996605

32. M.J. Dong, G.K. Hu, A. Diboine, D. Moulin, C. Prioul, J. Phys. IV France 3, 643- 648 (1993). doi: 10.1051/jp4:19937103

33. F. Rabold, M. Kuna, Comput. Mat. Sci 32, 489497 (2005). doi: 10.1016/j.commatsci.2004.09.016

34. D.O. Fernandino, A.P. Cisilino, R.E. Boeri, Mech. Mat. 83, 110-121 (2015). doi: 10.1016/j.mechmat.2015.01.002

35. F.D. Carazo, S.M. Giusti, A.D. Boccardo, L.A. Godoy, Comput. Mat. Sci. 82, 378-390 (2014). doi: 10.1016/j.commatsci.2013.09.044

36. F. Carazo, S. Giusti, A. Boccardo, P. Dardati, L Godoy, Mec. Comput. 30, 611-629 (2011)

37. V. Di Cocco, D. Iacoviello, F. Iacoviello, A. Rossi, Pro. Eng. 109, 135-143 (2015). doi:

10.1016/j.proeng.2015.06.223
38. F. Klubberg, P. Beiss, Effizienzsteig. d. innov. Werkstofft: Wkstfftag '95, 777-780 (1995)

39. Chen, G, Strength Prediction of Particulate Reinforced Metal Matrix Composites (Shaker, Aachen, 2016)

40. G. Chen, A. Bezold, C. Broeckmann, Comp. Struct. 189, 330-339 (2018). doi: 10.1016/j.compstruct.2018.01.005

41. P. Suquet, Elements of homogenization for inelastic solid mechanics (Universite des Sciences et Technique du Languedoc, 1985)

42. O. Pierard, Micromechanics of inclusionreinforced composites in elastoplasticity and elasto-viscoplasticity: modeling and computation. (Universite Catholique de Louvain, 2006)

43. J.A. König, Shakedown of elastic-plastic structures. Fundamental studies in engineering, 7 (Elsevier, Amsterdam, 1987)

44. A. François, H. Abdelkader, T. Le An, M. Said, P. Tao, J Glob Optim 37, 609-630 (2007). doi: 10.1007/s10898-006-9069-1

45. W. Bleck, Werkstoffkunde Stahl für Studium und Praxis (Mainz, Aachen, 2010)

46. F. Klubberg, M. Hempen, H. Schäfer, P. Beiss, 7th Ams. Symp. Wrld. Dyn. Tes., 318-342 (2000)

47. A. Velichko, F. Mücklich, PM 43, 192-207 (2006) doi: $10.3139 / 147.100297$ 